\title{
More evidence for an admixture of the Hyles euphorbiae complex's main lineages in Mediterranean Europe (Lepidoptera: Sphingidae)
}

\author{
Michael B. MENDE ${ }^{1,2,3}$ and AnNA K. HUNDSDOERFER ${ }^{2,3}$ \\ ${ }^{1}$ Georg-August-Universität Göttingen, Johann-Friedrich-Blumenbach-Institut für Zoologie und Anthropologie, \\ Abteilung für Morphologie, Systematik und Evolutionsbiologie, Berliner Str. 28, D-37073 Göttingen, Germany \\ ${ }^{2}$ Senckenberg Naturhistorische Sammlungen Dresden, Museum für Tierkunde, Königsbrücker Landstr. 159, D-01109 Dresden, \\ Germany \\ ${ }^{3}$ Biodiversität und Klima Forschungszentrum (BiK-F), Senckenberganlage 25, D-60325 Frankfurt am Main, Germany; \\ e-mails: michael.mende@entomologie.de; ahundsdoerfer@senckenberg.de
}

Key words. Lepidoptera, Sphingidae, Hyles euphorbiae complex, Hyles cretica, historical DNA, mtDNA, biogeography, island endemic, Aegean Islands, hybridization, introgression

\begin{abstract}
The populations of the Hyles euphorbiae complex on Crete and the Dodecanese Islands have been described as an endemic species, Hyles "cretica". Alternatively, they are thought to be of hybrid origin from the complex's two main lineages, the European $H$. euphorbiae and African H. tithymali, because of their intermediate morphology. That they belong to a discrete taxon is supported by the fact that all the recent samples analyzed belong to an endemic mitochondrial haplotype cluster; however, this cluster is so close to the tithymali cluster that it might also be regarded as a sublineage of H. tithymali. Similarly, southern Italy is currently dominated by another distinct tithymali-related cluster, named "italica". However, sequencing of historic museum specimens, which were collected in Italy during the past century, revealed that mitochondrial haplotypes of $H$. euphorbiae were once present throughout the entire range of "italica" thus contradicting a long-term isolated evolution of a southern Italian refugial entity. In the present study we likewise sequenced the few available historic specimens from the Aegean Islands and also found a specimen from Crete which belonged to the euphorbiae cluster. In conclusion, southern Italian and southern Aegean populations both appear to be dynamic hybrid mixtures of the European H. euphorbiae and African H. tithymali, rather than separately evolved taxa.
\end{abstract}

\section{INTRODUCTION}

Spurge hawk moths are a polymorphic species complex, widely distributed across the Western Palaearctic. Within the Hyles euphorbiae complex sensu stricto (HEC; for definition see Hundsdoerfer et al., 2009a) geographic differences in adult and especially larval colour patterns provoked the description of several taxa, among them two Mediterranean island endemics: H. cretica Eitschberger, Danner \& Surholt 1998 on Crete and the Dodecanese Islands and H. sammuti Eitschberger, Danner \& Surholt 1998 on Malta. However, because of their intermediate colour patterns, other authors (e.g. Meerman \& Smid, 1988; Pittaway, 1993; Hundsdoerfer et al., 2011a) considered these Mediterranean populations to be natural hybrid populations between the two main lineages of the HEC, namely H. euphorbiae (Linneaus, 1758), which is distributed across large parts of temperate Europe and Asia, and H. tithymali (Boisduval, 1832), which inhabits Macaronesian archipelagos, Magreb and an isolated area in Yemen. H. euphorbiae larvae typically have two large eyespots per segment and a lateral coloured wedge on the posterior part of each segment while $H$. tithymali typically lacks the lower eyespots and has a yellow-coloured lateral band across the segments connecting the upper row of eyespots. Southern Italian, Maltese and southern Aegean larvae have a yellow-sprinkled band and the lower eyespots are typically reduced in size but not completely absent; they also remarkably resemble captive bred hybrids between H. euphorbiae and H. tithymali (Harbich, 2009). Moreover, the costal margin of the adult forewing, which is typically bright in $H$. euphorbiae and generally darker and broader in $H$. tithymali, is mostly intermediately darkened in Mediterranean populations.
The validity of the Maltese $H$. "sammuti" has further been challenged based on mitochondrial markers, since it is mainly composed of a mixture of the euphorbiae and tithymali haplotype clusters (Hundsdoerfer et al., 2005a; 2009a; 2011b). In contrast, the validity of $H$. "cretica" is surprisingly corroborated by a distinct haplotype cluster, which is endemic to the southern Aegean Islands and currently the only cluster found there. Although this cluster is very close to the tithymali cluster and might thus be regarded as a sublineage of $H$. tithymali (see discussion for details), its distinctiveness suggests a long-term isolated evolution in a southern Aegean refuge during and since at least the last glaciation (Hundsdoerfer et al., 2011b). Similarly, the southern Italian population appeared to constitute a distinct entity based on another distinct tithymali-related haplotype cluster named "italica" (Hundsdoerfer et al., 2011b) and adult and larval morphology (e.g. Staudinger, 1885; deFreina \& Geck, 2002; Hundsdoerfer et al., 2011a). However, the two mitochondrial clusters of H. euphorbiae, named euphorbiae and "enigmatica", were once present throughout the entire range of "italica" at the beginning of the twentieth century, which indicates that the present-day prevalence of "italica" in southern Italy does not reflect a longtime separated taxon (Mende \& Hundsdoerfer, 2013). The disappearance of $H$. euphorbiae haplotypes from southern Italy was attributed to genetic drift, promoted by habitat destruction and fragmentation, and to the spreading of supposedly better warmadapted "italica" populations, promoted by recent climate warming. Since the HEC population on the southern Aegean Islands experienced habitat destruction (e.g. Tzatzanis et al., 2003) and climate change (Founda \& Giannakopoulos, 2009) in a similar way throughout the twentieth century, we also decided to investigate the biogeographical integrity of cretica. 
TABLE 1. Data on museum specimens collected on the Aegean Islands that were analyzed in this study.

\begin{tabular}{cccccc}
\hline MTD\# & Museum & Locality & Date; collector/ collection & DNA extr. & Mt-lineage \\
\hline 7304 & ZSM & [Greece,] Rhodos, Kalithe[a] & 16.vi.1983, e.1.; H. Peichl & leg & cretica \\
7305 & ZSM & [Greece,] Rhodos, Kalithe[a] & 10.vi.1983, e.1.; H. Peichl & leg & cretica \\
7364 & MWM & [Greece,] Kreta & 16.x.1987, e.1.; L. Ditgens & leg & euphorbiae \\
7368 & MWM & [Greece,] Rhodos & 05.xiii.1934 & leg & cretica \\
7369 & MWM & [Greece,] Rhodos & 12.vii.1939; Eberhardt & leg & cretica \\
7968 & NMH & Greece, Rhodos, Trianta & 22.ix.1963; M. Rothschild, M. Walker, T. Clay & abdomen & cretica \\
8003 & NMH & GR[eece], Lesbos, Lepetimnos & 29.vi.1975, e.1.; Coll. Cadiou & abdomen & euphorbiae \\
8023 & NMH & Greece, Rhodes, Ixos, Hotel Miramare & 09.-21.v.1964; Coll. Rothschild & abdomen & cretica \\
8024 & NMH & Greece, Rhodes, Ixos, Hotel Miramare & 09.-21.v.1964; Coll. Rothschild & abdomen & cretica \\
\hline
\end{tabular}

MTD\# - voucher number in the tissue catalogue of the Museum für Tierkunde, Dresden; Museum - source of the sample (ZSM = Zoologische Staatssammlung, Munich; MWM - Museum Witt, Munich; NMH - Natural History Museum, London); Locality - data in square brackets = completed by inference; DNA extr. - DNA extraction from leg sample (leg) or abdominal tissue macerate (abdomen; see Hundsdoerfer \& Kitching, 2010).

\section{MATERIAL AND METHODS}

Historic specimens of $H$. "cretica" are significantly less frequent in Central European museum collections than samples from southern Italy (own observations of MM), which could be attributed to less collecting activities in this region. For instance, the HEC population on Crete was first described morphologically in 1988 and its local occurrence briefly noticed only twice before that (Meerman \& Smid, 1988). Nevertheless, we were able to obtain tissue samples from nine historic specimens from the Aegean Islands (Table 1). Morphological features of the voucher moths are in accordance with those of $\mathrm{H}$. "cretica" (see inset photograph in Fig. 1), except for the specimen from Lesbos (Table 1: \#8003), which resembles $H$. euphorbiae.

We performed two DNA extractions per specimen using the Agowa sbeadex Forensic Kit (Agowa Genomics) and subsequent multiplex/simplex PCRs in a dedicated clean laboratory along with negative and positive controls in each step for authentication (for protocol details and primers see Mende \& Hundsdoerfer, 2013). Amplification products of three targeted fragments of mitochondrial COI/II genes (277, 280 and 237 bp in length) were sequenced in both directions on an ABI 3130xl (Applied Biosystems) and the sequences aligned with BioEdit 7.0.9.0 (Hall, 1999). We were able to amplify the three fragments from every historic specimen (Supplementary file S1) enabling unambiguous assignment to a mitochondrial haplotype cluster using the polymorphic sites (see Mende \& Hundsdoerfer, 2013) and a median-joining network calculated using Network 4.5.1.0 (Fluxus Technology; Fig. 1A); for reference, the alignment included 60 haplotypes which resulted from the reduction of the contiguous $\mathrm{COI} / \mathrm{II}$ sequences of all 356 recent HEC specimens published by Hundsdoerfer et al. (2011b) to the concerning three fragments (see Mende \& Hundsdoerfer, 2013; Supplementary file S1). No conflicts in cluster assignment between fragments of each historic specimen and no nucleotide misincorporations were detected.

\section{RESULTS AND DISCUSSION}

Sequences of all seven specimens from Rhodes caught between 1934 and 1983 (Table 1) were identical to the sequence of the cretica haplotype that was recently found on all the southern Aegean Islands sampled, i.e. Crete, Rhodes, Samos and Chalki (Fig. 1; Supplementary file S1; sequence identical to base positions $110-345,1116-1351$ and $1931-2123$ of e.g. GB Acc. No. FR839597). In accordance with its morphological features, the specimen from Lesbos caught in 1975 (Table 1: \#8003) carried a euphorbiae haplotype, which was recently recorded in Bulgaria (Fig. 1; Supplementary file S1; sequence identical to positions as above of e.g. GB Acc. No. FR839377). It is possible that cretica haplotypes never reached this northern Aegean Island and it was only populated by euphorbiae from the close by Turkish mainland. Most interestingly, the specimen collected in 1987 on Crete (Table 1: \#7364) carried a common euphorbiae haplotype that is currently widely distributed across Europe from Eastern Spain to Greece (Fig. 1; Supplementary file S1; sequence identical to positions as above of e.g. GB Acc. No. FR839385). Accordingly, the euphorbiae cluster was present in the most remote and supposedly original refuge area of the endemic cretica haplotype cluster, which is similar to the past admixture of euphorbiae and "italica" in southernmost Italy (see Mende \& Hundsdoerfer, 2013). This admixture consequently contradicts the hypothesis of a welldefined long-term separately evolved entity $H$. "cretica" as suggested by recent mitochondrial phylogeography alone (Hundsdoerfer et al., 2011b). In addition, a supposed prezygotic isolation barrier between $H$. "cretica" and other HEC populations appears not to exist: based on few ex situ observations, Meerman \& Smid (1988) state that mating of specimens from Crete takes place in early morning whereas $H$. euphorbiae copulates in the evening before midnight (Harbich, 1976). However, other observations on captive $H$. "cretica" record copulations already three hours after sunset (H. Harbich, pers. comm.), which contradicts the supposed temporal isolation.

In a wider sense, the taxon $H$. tithymali can be seen as being represented by the haplotype clusters tithymali, "italica" and cretica since they are each only differentiated by a $0.28 \%$ minimal sequence difference and thus very closely cluster together (five base changes in 1805 bp; see Hundsdoerfer et al., 2011b; note that the network in Fig. 1 is biased since it is reduced to the three short fragments that best discriminate the clusters). Consequently, the southern Aegean population, like the southern Italian population, can be interpreted as a hybrid mixture of the HEC's two main lineages, the African $H$. tithymali (represented by the cretica mitochondrial cluster) and European H. euphorbiae (represented by the euphorbiae haplotype recorded in this study), corroborating the hybrid origin hypothesis that was previously postulated based on the intermediate morphological characters of these Mediterranean populations (see introduction). An investigation of nuclear microsatellite markers (Hundsdoerfer et al., 2009b; Mende et al., 2011) is likely to further elucidate this hypothesis (Mende et al., in prep.).

In conclusion, the finding of a euphorbiae haplotype on Crete among the dominant tithymali-derived cretica cluster suggests that as described for the southern Italian population a similar dynamic admixture of the two main lineages of the HEC occurred 


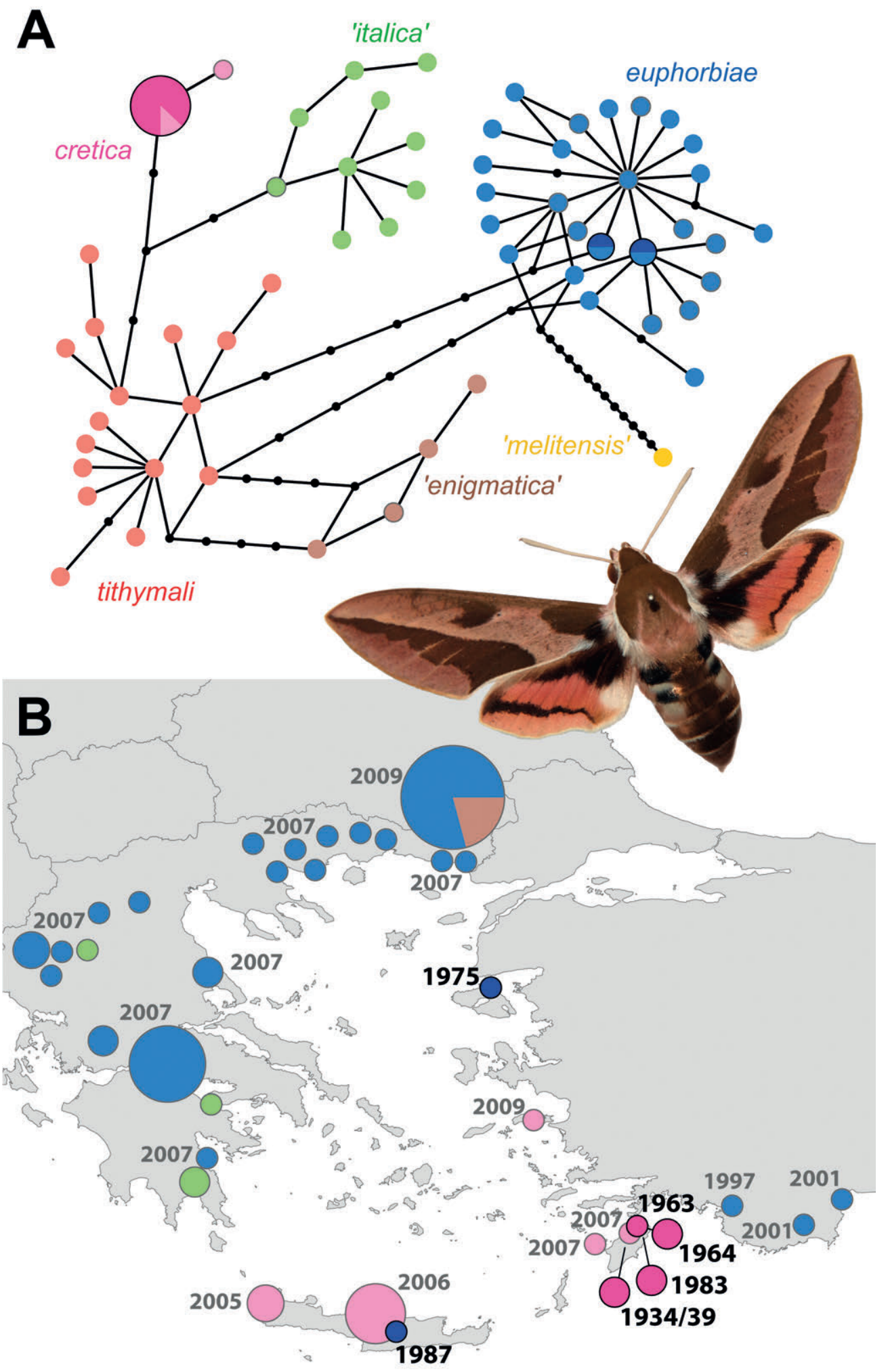

Fig. 1. Haplotype network for partial sequences of COI/II genes and distribution of the HEC's mitochondrial haplotype clusters in the Aegean region. A - Haplotype network of historical samples (marked by bold colour and black border) and haplotypes of recent Mediterranean HEC samples from the data set of Hundsdoerfer et al. (2011b; reduction to the three fragments used in the present study resulted in 60 haplotypes) (marked by faint colour; haplotypes with grey border occur in Bulgaria, Greece and/or Turkey). General colour coding and cluster names follow coding of mitochondrial lineages in Hundsdoerfer et al. (2011b) (clusters "enigmatica" and "melitensis" have not yet been detected in the area covered by this study but occur sympatrically among the euphorbiae lineage throughout Western Eurasia or solely on Malta, respectively); black nodes represent one substitution each. Inset: historic specimen \#7364 from Crete. B - Distribution of the HEC's haplotype clusters in the Aegean region. Size of the pie charts corresponds to the number of individuals per locality; year of collection is given. Colours of clusters correspond to Fig. 1A; recent samples in faint colours (1997-2009; data from Hundsdoerfer et al., 2011b), historic samples in bold (1934-1987). 
(and probably still occurs) on the southern Aegean islands (see Mende \& Hundsdoerfer, 2013).

ACKNOWLEDGEMENTS. The present study was conducted at the DNA laboratory of the "Senckenberg Natural History Collections Dresden" and financially supported by the research funding programme "LOEWE - Landes-Offensive zur Entwicklung Wissenschaftlich-ökonomischer Exzellenz" of Hesse's Ministry of Higher Education, Research and the Arts via the Biodiversity and Climate Research Centre (BiK-F), Frankfurt am Main. Furthermore, we like to thank I. Kitching, G. Martin (BMNH), A. Hausmann (ZSM) and W. Speidel (MWM) for permission to collect samples and two anonymous reviewers for valuable comments on the manuscript.

\section{REFERENCES}

Danner F., Eitschberger U. \& Surholt B. 1998: Die Schwärmer der westlichen Palaearktis. Bausteine zu einer Revision (Lepidoptera: Sphingidae). - Herbipoliana 4: 1-368.

De Freina J. \& Geck M. 2002: Bemerkungen zum Hyles euphorbiae (Linnaeus, 1758)-Komplex, Beschreibung der Larvalstadien sizilianischer "euphorbiae" und die Frage, ob diese Populationen taxonomisch neu zu bewerten sind: Hyles sammuti Eitschberger, Danner \& Surholt, 1998 subspec. nov.? Atalanta 33: 403-409.

Founda D. \& Giannakopoulos C. 2009: The exceptionally hot summer of 2007 in Athens, Greece - A typical summer in the future climate? - Glob. Planet. Change 67: 227-236.

HALL T.A. 1999: BioEdit: a user-friendly biological sequence alignment editor and analysis program for Windows 95/98/NT. — Nucl. Acids Symp. Ser. 41: 95-98.

HaRbich H. 2009: Anmerkungen zur Wolfsmilchschwärmerpopulation (Hyles euphorbiae (Linnaeus, 1758)-Komplex) von Malta (Lepidoptera: Sphingidae). - Entomol. Z. 119(2): 51-58.

Hundsdoerfer A.K., Kitching I.J. \& WinK M. 2005: A molecular phylogeny of the hawkmoth genus Hyles (Lepidoptera: Sphingidae, Macroglossinae). - Mol. Phylogen. Evol. 35: 442-458.

Hundsdoerfer A.K., Rubinoff D., Attie M., Wink M. \& KitchING I.J. 2009a: A revised molecular phylogeny of the globally distributed hawkmoth genus Hyles (Lepidoptera: Sphingidae), based on mitochondrial and nuclear DNA sequences. - Mol. Phylogen. Evol. 52: 852-865.

Hundsdoerfer A.K., Sanetra M., Corbeil D. \& Stuckas H. 2009b: Eleven hawkmoth microsatellite loci of Canary Island Hyles tithymali (Lepidoptera). — Conserv. Genet. Res. 2: 241244.
HundSDOERFER A.K. \& Kitching I.J. 2010: A method for improving DNA yield from century-plus old specimens of large Lepidoptera while minimizing damage to external and internal abdominal characters. - Arthr. Syst. Phylogen. 68: 151-155.

Hundsdoerfer A.K., Mende M.B., Harbich H., Pittaway A.R. \& Kitching I.J. 2011a: Larval pattern morphotypes in the Western Palaearctic Hyles euphorbiae complex (Lepidoptera: Sphingidae: Macroglossinae). — Insect Syst. Evol. 42: 41-86.

Hundsdoerfer A.K., Mende M.B., Kitching I.J. \& Cordellier M. 2011b: Taxonomy, phylogeography and climate relations of the Western Palaearctic spurge hawkmoth (Lepidoptera, Sphingidae, Macroglossinae). - Zool. Scr. 40: 403-417.

Meerman J.C. \& Smid G. 1988: Der Hyles euphorbiae-Komplex; die Wolfsmilchschwärmer von Kreta (Lepidoptera: Sphingidae). - Entomol. Z. 98(12): 161-176.

Mende M.B., Stuckas H. \& Hundsdoerfer A.K. 2011: Eight new microsatellite loci of the Western Palearctic Hyles euphorbiae complex (Lepidoptera, Sphingidae). — Ann. Zool. Fenn. 48: 142-146.

Mende M.B. \& Hundsdoerfer A.K. 2013: Mitochondrial lineage sorting in action - historical biogeography of the Hyles euphorbiae complex (Sphingidae, Lepidoptera) in Italy. - BMC Evol. Biol. 13: 83. doi:10.1186/1471-2148-13-83

Pittaway A.R. 1993: The Hawkmoths of the Western Palaearctic. Harley Books, Colchester, 240 pp.

STAUDINGER O. 1885: Deilephila Euphorbiae L. var. Grentzenbergi Stgr., und eine neue Anthocharis. — Entomol. Nachr. 1: 10.

Tzatzanis M., WrbKa T. \& Sauberer N. 2003: Landscape and vegetation responses to human impact in sandy coasts of Western Crete, Greece. - J. Nat. Conserv. 11: 187-195.

Received January 10, 2014; revised and accepted May 26, 2014 Prepublished online August 15, 2014

Supplementary file S1 (http://www.eje.cz/2014/065/S01.fas). Alignment of three sequence fragments of COI/II genes each from historic Aegean specimens and recent reference sequences. Sequence fragment "B" from base position 1-236, fragment "H" from 237-472, fragment "L" from 473-665. Sequence identifier corresponds to voucher number in the invertebrates tissue catalogue of the Museum für Tierkunde, Dresden (also see Table 1 for historic Aegean specimens); identifiers of exemplary recent reference sequences from Hundsdoerfer et al. (2011b) are preceded by a letter for the mitochondrial haplotype cluster $(\mathrm{A}=$ euphorbiae, $\mathrm{B}=$ tithymali, $\mathrm{C}=$ "melitensis", $\mathrm{D}=$ "italica", $\mathrm{E}=$ cretica, $\mathrm{F}=$ "enigmatica") and a haplotype number followed by the corresponding exemplary GenBank accession number. 